\title{
Relationship between selected anthropometric variables, cardiovascular risk factors and endurance walk in non-insulin dependent diabetic participants
}

\author{
Oluwafemi Oluwasegun ${ }^{1}$, Adeyanju Solomon Adekunle ${ }^{2}$, Onigbinde Ayodele Teslim ${ }^{3}$ \\ ${ }^{1}$ Department of Physiotherapy, State Hospital, Asubiaro, Osun State, Nigeria \\ ${ }^{2}$ Department of Physical \& Health Education, Obafemi Awolowo University, Ile-Ife, Osun State, Nigeria \\ ${ }^{3}$ Department of Medical Rehabilitation, College of Health Sciences, Obafemi Awolowo University, Ile-Ife, Osun State, Nigeria
}

Email address:

segunoluwafemi@yahoo.com (Oluwafemi O.)

\section{To cite this article:}

Oluwafemi Oluwasegun, Adesanju Solomon Adekunle, Onigbinde Ayodele Teslim. Relationship between Selected Anthropometric Variables, Cardiovascular Risk Factors and Endurance Walk in Non-Insulin Dependent Diabetic Participants. American Journal of Health Research. Special Issue: Supplementary Prescribing in Nigeria: A Needy Concept to Promote Clinical Physiotherapy Practice.

Vol. 2, No. 5-1, 2014, pp. 61-66. doi: 10.11648/j.ajhr.s.2014020501.22

\begin{abstract}
There appears to be an increasing number of patients with cardiovascular disorders and diabetes but there is paucity of data on likely factors which are related to these disorders. The primary purpose of this study was to examine the relationship between selected anthropometric variables, cardiovascular disease risk factors and cardiovascular response to endurance walk in participants with diabetics. Sixty participants each, with non-insulin dependent and without diabetes were recruited for this study. The weight, height, Body Mass Index, Waist Circumference, Hip Circumference, Sagittal Abdominal Diameter, Waist to Hip Ratio, Waist to Height Ratio, \%BF-Percent Body Fat, blood pressure, fasting blood sugar, and distance covered in 6-minute walk test; and other selected adipose tissue variables were measured for each participant while percentage body fat was computed. The descriptive statistics and student $\mathrm{t}$-test were used to analyze the data. The result showed that the age, weight, BP, PR, BMI, WC, WTH, \%BF and SAD of the diabetics were significantly higher than that of the non-diabetics $(\mathrm{P}<0.05)$. There was no significant difference between all the selected cardiovascular risk factors between male and female but the distance covered by male was significantly higher than that of female $(t=1.89, p=0.006)$. There was significant relationship between systolic BP and BMI $(r=0.27, p=0.04)$ and similar trend was observed for between FBS and WHR $(r=$ $0.26, \mathrm{p}=0.05)$. There was also significant relationship between distances covered during 6-minutes walk test, weight $(\mathrm{r}=-0.28$, $\mathrm{p}=0.03)$ and BMI $(\mathrm{r}=-0.29, \mathrm{p}=0.03)$. It was concluded that there was significant relationship between BMI, WHR, blood pressure and blood glucose. Similarly, there was significant difference in most of the anthropometric and adipose tissue variables of diabetics and non-diabetics.
\end{abstract}

Keywords: Cardiovascular Risks, Fasting Blood Sugar, Diabetes, Relationship

\section{Introduction}

Cardiovascular disorder is a disease affecting the heart and blood vessels. Cardiovascular disease (CVD) accounted for a large proportion of mortality in adults older than 45 years [1]. Also, the American Heart Association reported that CVD is the number one killer in the United States and that black women within the age range of 35-74 years, had a higher death rate $(72 \%)$ from coronary heart disease than white women. People with CVD tend to have reduced level of physical activity which also affects their fitness level [2].

Diabetes has been identified as one of the risk factors in the development of CVD [1]. People with type 2 diabetes have greater incidence of cardiovascular disease, and renal disease than the general population [3]. Mortality from cardiovascular diseases has been reported to also increase two to four fold in diabetics, over the general population [4, 5].

Van Itallie reported that a number of anthropometric measurable attributes of the human body could be related to risk of developing metabolic disorders and various illnesses [6]. He mentioned these attributes to include stature, relative weight, muscularity, frame size, various weights to height indices, waist circumference (in relation to chest or hip 
circumference), skin-fold thickness at various sites, and the overall pattern of distribution of subcutaneous fat.

Various studies have linked specific anthropometric variables to particular health problems. Wei et al reported that waist circumference have the highest correlation with body Weight, Body Mass Index (BMI), Waist Circumferences (WC) and Hip Circumferences (HC), Waist to Hip Ratio (WHR) and Waist to Height Ratio (WTH), [7]. There are various studies which had been done on relationship between diabetes, cardiovascular risk factors and anthropometric parameters but these are majorly in Europe, Asia and Caribbean island [6, 8, 9, 10, 11, 12]. There appears to be an increasing number of patients receiving treatments for cardiovascular disorders and diabetes but there is paucity of data to ascertain this speculation. Considering racial differences and lifestyle between Europeans, Asia and Africa, it will be difficult to generalize findings from previous studies, hence, the need to examine the relationship between anthropometric variables, cardiovascular disease risk factors and endurance walk of individual with diabetes. The primary purpose of this study was to examine the relationship between selected anthropometric variables, cardiovascular disease risk factors and cardiovascular response to endurance walk in participants with diabetics.

\section{Methodology}

\subsection{Subjects}

One hundred and twenty participants were recruited for this study. Sixty participants each, with and without noninsulin dependent diabetes receiving treatment at the out Patient department of 3 State Hospitals located at Osogbo, Ede and Ila-Orangun, Osun State, Nigeria volunteered to take part in the study.

\subsection{Research Design}

A case control groups design method was employed.

\subsection{Sampling Technique}

Subjects diagnosed by physicians of having non insulin dependent diabetics and had been receiving treatment were purposively selected for the test group while apparently healthy individual (those without diabetes or who were not in transition or intermediate state) were considered for the control group after evaluation of the Fasting Blood Glucose. The physically challenged, people with history of heart problem and known athletes were excluded from participating in the study.

Participants in the test group were recruited through balloting. Figures 1 to 60 were written on small pieces of paper with a number on each piece of paper, the pieces were folded along with other blank folded papers (without any number written on them). The participants were asked to pick a paper each. Those that picked the papers with numbers written on them were taken to be subjects for the groups.

\subsection{Research Instruments}

1. The instruments utilized for the study are: Glucometer (One Touch -Ultra easy model, LIFE SCAN Johnson and Johnson Company, 021-195), Mercury sphygmomanometer (Accoson Model), Stethoscope (UMEC S-223), Stadiometer, and weighing scale (Harson, H89 Light Green Model). Others are; an inelastic leather tape (Butterfly brand, China), Skin fold caliper (Cambridge Scientific International Maryland pat Number 3008239), Stop watch (Nokia 1208) and a relative plane hard surface of 30 meter long used by the participants for 6-minute walk test.

Prior to the main study, the reliability of the major instruments were established with a test-re-test interval of 2 weeks using 10 participants. The reliability co-efficient (r) for the mercury sphygmomanometer for systolic and diastolic blood pressure were 0.98, $(\mathrm{P}<0.05)$ and $0.90 \quad(\mathrm{P}<0.05)$ respectively while for the skin fold calipers was 0.88 , $(\mathrm{P}<0.005)$. The reliability co-efficient for the glucometer was found to be $0.98(\mathrm{P}<0.05)$.

\subsection{Procedure}

The age of the participants were taken by asking what their age was. Fasting blood sugar readings were taken from the participants before eating and when they had fasted for at least 8-hours. This was done by collecting a drop of blood sample through the use of a needle prick (a steriled lancet tip) on the thumb of each subject, the drop of blood was applied on a Glucometer (One Touch-Ultra easy model LIFESCANJohnson and Johnson Company, 021-195) the results obtained were recorded.

The following anthropometric variables; weight, height, $\mathrm{WC}, \mathrm{HC}, \mathrm{SAD}$ were then measured from each participant. Body weight and height were measured with subjects wearing light clothing and without shoe, cap or head gear. The weighing scale was adjusted to zero before body weight was measured; each participant was made to stand on the bathroom weighing scale, and the weight taken in kilograms $(\mathrm{kg})$. The measurement and collection of data was done at the Physiotherapy Departments of State Hospital Osogbo, Ede and Ila-Orangun.

\subsection{Anthropometric Measurements}

The height, in meters (m) was measured as the participant stood against a vertically graduated wall. A small ruler was placed on the head of the participant horizontally so that one end of the ruler touched the graduations on the wall, the point on the graduated wall where the small ruler met the graduated lines on the wall was noted and recorded as height of the participant BMI was calculated by dividing weight in kilogram with square of height in meters. $\left(\mathrm{Kg} / \mathrm{m}^{2}\right)$.

A non-elastic measuring leather tape was used to measure WC. Waist Circumference was taken from the narrowest circumference between the lowest rib and the top of the pelvis while the participant was standing. The measurement was taken at the end of a gentle expiration. The hip 
circumference was taken as the maximum extension of the buttocks, while the participant remained standing. The measuring tape was placed round the level of maximum extension of the buttock. The reading was taken in centimeter. SAD was taken as the greatest vertical height of the abdomen when the participant was lying supine. A paper cardboard of about $20 \mathrm{~cm}$ by $40 \mathrm{~cm}$ was placed on top of the abdomen when participant was in supine position, the zero end of the measuring tape was placed on top of the couch by the side of the abdomen to meet the end of the cardboard. The meeting point of the cardboard on the vertically placed measuring tape from the couch was taken as the SAD of the participant in centimeter. Body fat was estimated by measurement of skin fold thicknesses through skin folds calipers. The measurement was done by setting thumb and index finger approximately one centimeter above and below the appropriate measuring point, to form an "O" with the finger. The skin was pulled away from the body, the thickness of the skin pulled away from the body was then measured with skin fold calipers in $\mathrm{mm}$.

For male participants, region for skin fold measurement were chest, waist and anterior thigh, for females, the region for skin fold measurement were triceps, waist and hip. The formular for calculating the body fat are:

For women, $X=1.089733-0.0009245 \times$ (sum of the three folds $)+0.0000056 \times$ (sum of the three folds $)^{2}-0.00012828$ (age in years):

Body fat in $\%={ }^{495} / x-450$

For men, $X=1.1093800-0.0008267 \times$ (sum of the three folds $)+0.0000016 \times$ (sum of the three folds $)^{2}-0.0002574$ (age in years):

Body fat in $\%=495 / x-450$

The formula for calculating the body fat with the 3-folds formula was by Jackson and Pollock (2008) as adapted by Calculate Body Fat (Adipose) Rate. Computer was used to compute the $\%$ body fat.

\subsection{Measurement of Cardiovascular Variables}

Measurement of blood pressure was carried out with mercury sphygmomanometer of Accoson model and U-MEC S-223 Stethoscope. The measurement was carried out in accordance with the American Heart Association's recommendation as adopted by McAlister and Straus (2001). The subject was made to rest for a minimum of 10-minutes by sitting comfortably. The sphygmomanometer cuff with the bladder was wrapped round the arm of the subject; the earpiece of the stethoscope was put into my ear canal. The head of the stethoscope was placed over the brachial artery pulsation. The cuff was then inflated, the first and fifth Korotkoff sound was taken during deflation and recorded as the systolic and diastolic blood pressures in $\mathrm{mm} \mathrm{Hg}$. Pulse Rate was estimated through radial pulse. The number of pulse was taken for 10 seconds and multiplied by 6 to have numbers of pulse per minutes.

\subsection{Six Minutes Walk Test}

Administration of Six Minute Walk test was carried out when the subjects had been informed of the nature of the walking. Each participant was asked to walk at his or her own convenient pace on a relative plane ground of 30 meter long marked out for the walking exercise. The walking started at one-end of the area marked out in a straight line to the other end. Each participant was asked to walk 'to and fro' for six minutes along the area marked out. A stop watch was used to time the participant during the walking so that the distance covered at the end of sixth minute was measured. Before the walk, resting BP and pulse rate of the participant was taken; this was done after each participant sat comfortably for at least ten minutes. After sixth minutes, the participant was asked to stop and sat in a chair from where the post exercise BP and pulse rate were taken. The BP and pulse rate were taken at the place marked out for the six minutes walk test. Distance covered was also calculated in meters.

\subsection{Statistical Analysis}

The data were analyzed using descriptive statistics of mean, standard deviation and frequency distribution. Also, t-test was used to compare value obtained for the diabetic and non diabetic participants. Test of relationship between anthropometric variables and cardiovascular variables was done using pearson product moment correlation.

\section{Results}

There are 30 male $(50 \%)$ and 30 female $50 \%$ among the diabetic subjects (group 1) while there are 38 male (63.3\%) and 22 female $(33.7 \%)$ in the non-diabetic group (group 2), (Table 1). The result comparing the physical and anthropometric Parameters is also presented in table 1.

Table 1. Physical and Anthropometric Parameters of the groups

\begin{tabular}{llllll}
\hline & & Mean & SD & t-value & P \\
\hline \multirow{2}{*}{ Age (year) } & Diabetic & 60.35 & 7.35 & & \\
& Non- Diabetic & 55.93 & 6.61 & 3.46 & $0.001^{*}$ \\
Weight (Kg) & Diabetic & 75.88 & 13.00 & & \\
& Non- Diabetic & 67.52 & 10.51 & 3.88 & $0.01^{*}$ \\
Height (m) & Diabetic & 1.65 & 0.06 & & \\
& Non- Diabetic & 1.65 & 0.06 & 0.37 & 0.71 \\
\hline
\end{tabular}

*Significant at $\mathrm{P}<0.05$

Table 2 presents the result of analysis comparing the Body Mass Index and other adipose tissue variables of diabetic and non-diabetic participants. Table 3 presents the result of comparison of the physical attributes of the male and female diabetic subjects. The result in showed that the mean BMI for the male diabetic participants was $27.32 \mathrm{~kg} / \mathrm{m}^{2}$ while that of female diabetic participants was $27.96 \mathrm{~kg} / \mathrm{m}^{2}$. Table 4 also presents the outcome of comparison of adipose tissue variables of the 2 groups.

Table 5 presents the analysis result comparing the cardiovascular variables of diabetic and non-diabetic participants. In all the cardiovascular variables measured, the diabetic group had higher mean values than the non-diabetic 
group. Result in table 6 shows mean value of FBS for male diabetics to be $134.73 \pm 50.6 \mathrm{mg} / \mathrm{dl}$ while that for female diabetics was $124.43 \pm 40.06 \mathrm{mg} / \mathrm{dl}$. Male diabetics FBS was higher by $10.30 \mathrm{mg} / \mathrm{dl}$. The results showed no significant difference in the selected

Table 2. Comparison of the Body Mass Index and other adipose tissue variables of diabetic and non-diabetic participants

\begin{tabular}{llllll}
\hline & & Mean & SD & t-value & P \\
\hline \multirow{2}{*}{ BMI $(\mathrm{kg} / \mathrm{m} 2)$} & Diabetic & 27.64 & 4.62 & & \\
& Non- Diabetic & 24.77 & 3.54 & 3.83 & $0.001^{*}$ \\
WC $(\mathrm{cm})$ & Diabetic & 96.40 & 10.46 & & \\
& Non- Diabetic & 89.65 & 10.46 & 3.54 & $0.001^{*}$ \\
HC $(\mathrm{cm})$ & Diabetic & 104.09 & 16.53 & & \\
& Non- Diabetic & 99.63 & 10.69 & -1.76 & 0.08 \\
SAD $(\mathrm{cm})$ & Diabetic & 22.12 & 2.83 & & \\
& Non-Diabetic & 19.71 & 2.87 & 4.63 & $0.001^{*}$ \\
WHR & Diabetic & 0.91 & 0.04 & & \\
& Non- Diabetic & 0.90 & 0.03 & 1.24 & 0.22 \\
WTH & Diabetic & 58.40 & 6.43 & & \\
& Non- Diabetic & 54.64 & 5.79 & 3.36 & $0.01^{*}$ \\
\%BF & Diabetic & 23.22 & 2.69 & & \\
& Non- Diabetic & 20.88 & 5.77 & 2.84 & $0.005^{*}$ \\
\hline
\end{tabular}

*Significant at $\mathrm{P}<0.05$, BMI-Body Mass Index, WC-Waist Circumference, HC-Hip Circumference, SAD-Saggital Abdominal Diameter, WHR-Waist to Hip Ratio, WTH-Waist to Height Ratio, \%BF-Percent Body Fat.

Table 3. The result of comparison of the physical attributes of the male and female diabetic subjects

\begin{tabular}{llllll}
\hline & & Mean & SD & t-value & P \\
\hline \multirow{2}{*}{ Age (year) } & Male & 60.93 & 7.22 & & \\
& Female & 59.77 & 7.54 & 0.61 & 0.54 \\
Weight $(\mathrm{kg})$ & Male & 77.47 & 13.48 & & \\
& Female & 74.30 & 12.53 & 0.94 & 0.35 \\
Height $(\mathrm{m})$ & Male & 1.68 & 0.06 & & \\
& Female & 1.62 & 0.04 & 4.36 & $0.01^{*}$ \\
\hline
\end{tabular}

*Significant at $\mathrm{P}<0.05$

Table 4. Comparison of adipose tissue variables of the 2 groups

\begin{tabular}{llllll}
\hline & & Mean & SD & t-value & P \\
\hline \multirow{2}{*}{ BMI $(\mathrm{kg} / \mathrm{m} 2)$} & Male & 27.32 & 4.45 & & \\
& Female & 27.96 & 4.83 & -0.53 & 0.6 \\
WC $(\mathrm{cm})$ & Male & 97.10 & 10.10 & & \\
& Female & 95.70 & 10.93 & 0.52 & 0.61 \\
HC $(\mathrm{cm})$ & Male & 102.45 & 20.54 & & \\
& Female & 105.73 & 2.78 & -0.77 & 0.45 \\
SAD & Male & 21.68 & 2.78 & & \\
& Female & 22.55 & 2.86 & -1.19 & 0.24 \\
WHR & Male & 0.92 & 0.04 & & \\
& Female & 0.91 & 0.04 & 0.13 & 0.90 \\
WTH & Male & 57.89 & 6.18 & & \\
& Female & 58.90 & 6.74 & -0.6 & 0.55 \\
\%BF & Male & 23.33 & 3.44 & & \\
& Female & 23.10 & 1.70 & 0.32 & 0.75 \\
\hline
\end{tabular}

There was significant relationship between systolic BP and BMI. There was also significant relationship between FBS and WHR (Table 7). Table 7 presents other results on relationship between other anthropometric Variables and cardiovascular Risk Factors (Blood pressure and blood glucose) of the diabetic group. Table 8 presents the result of correlation analysis of physical attributes with distance covered during six minutes walk test. There was significant relationship between distances covered during 6-minutes walk test, weight and BMI.

Table 5. Results comparing the cardiovascular variables of diabetic and non-diabetic participants

\begin{tabular}{llllll}
\hline & & Mean & SD & t-value & P \\
\hline FBS (mg/dl) & Diabetic & 129.58 & 45.59 & & \\
& Non - Diabetic & 76.35 & 14.58 & 8.61 & $0.001^{*}$ \\
SBP1 & Diabetic & 137.47 & 11.11 & & \\
$(\mathrm{mmHg})$ & Non- Diabetic & 130.83 & 10.11 & 3.35 & $0.001^{*}$ \\
DBP1 & Diabetic & 87.27 & 5.42 & & \\
$(\mathrm{mmHg})$ & Non- Diabetic & 82.17 & 9.13 & 3.72 & $0.001^{*}$ \\
SBP2 & Diabetic & 142.60 & 8.34 & & \\
$(\mathrm{mmHg})$ & Non- Diabetic & 134.28 & 9.67 & 5.01 & $0.001^{*}$ \\
DBP2 & Diabetic & 89.30 & 4.84 & & \\
$(\mathrm{mmHg})$ & Non- Diabetic & 85.30 & 6.32 & 4.06 & $0.01^{*}$ \\
PR1 (beats / & Diabetic & 79.68 & 6.24 & & \\
min) & Non- Diabetic & 77.17 & 6.88 & 2.07 & $0.04^{*}$ \\
PR2 (beats / & Diabetic & 99.73 & 8.50 & & \\
min) & Non- Diabetic & 90.72 & 9.41 & 5.44 & $0.01^{*}$ \\
& Diabetic & 213.42 & 25.43 & & \\
Dist (m) & Non- Diabetic & 234.50 & 21.92 & -4.80 & $0.001^{*}$ \\
\hline
\end{tabular}

*Significant at $\mathrm{P}<0.05$, FBS-fasting blood sugar, $\mathrm{SBP}_{1}$-resting systolic blood pressure, $\mathrm{DBP}_{1}$-resting diastolic blood pressure, $\mathrm{SBP}_{2}$ - post six minute walk test systolic blood pressure, $\mathrm{DBP}_{2}$ - post six minute walk test diastolic blood pressure, $\mathrm{PR}_{1}$ - resting pulse rate, $\mathrm{PR}_{2}$ - post six minute walk test pulse rate, Dist- distance covered during 6 minutes walk test

Table 6. Comparison of the FBS, blood pressure and distance covered along gender distribution

\begin{tabular}{llllll}
\hline & & Mean & SD & t-value & P \\
\hline \multirow{2}{*}{ FBS (mg/dl) } & Male & 134.73 & 50.69 & & \\
& Female & 124.43 & 40.06 & 0.87 & 0.39 \\
SBP1 (mmHg) & Male & 137.00 & 11.35 & & \\
& Female & 137.93 & 11.84 & -0.31 & 0.76 \\
DBP1 (mmHg) & Male & 86.47 & 5.55 & & \\
& Female & 88.07 & 5.27 & -1.15 & 0.26 \\
SBP2 (mmHg) & Male & 141.93 & 7.74 & & \\
& Female & 143.21 & 8.88 & -0.99 & 0.33 \\
DBP2 (mmHg) & Male & 88.48 & 4.77 & & \\
& Female & 90.07 & 6.76 & -1.68 & 0.10 \\
Pulse1 (beat/ min) & Male & 81.10 & 6.13 & & \\
& Female & 77.89 & 6.14 & 1.76 & 0.08 \\
& Male & 101.52 & 8.38 & & \\
Pulse2 (beat/min) & Female & 97.29 & 8.22 & 1.61 & 0.11 \\
& Male & 218.79 & 17.67 & & \\
Dist (m) & Female & 207.75 & 25.36 & 1.89 & 0.006 \\
\hline
\end{tabular}

Cardiovascular parameters of male and female but the distance covered by male was significantly higher than that of female $(t=1.89, \mathrm{p}=0.006)$. 
Table 7. Relationship between fasting blood sugar and selected anthropometric parameters

\begin{tabular}{lllll}
\hline & & $\begin{array}{l}\text { FBS } \\
(\mathbf{m g} / \mathbf{d l})\end{array}$ & $\begin{array}{l}\text { SBP } \\
(\mathbf{m m H g})\end{array}$ & $\begin{array}{l}\text { DBP } \\
(\mathbf{m m H g})\end{array}$ \\
\hline BMI & Pearson correlation & 0.13 & $0.27^{*}$ & 0.18 \\
$(\mathrm{~kg} / \mathrm{m} 2)$ & Sig (2-tailed) & 0.34 & 0.04 & 0.17 \\
& $\mathrm{~N}$ & 60 & 60 & 60 \\
& Pearson correlation & 0.10 & -0.20 & -0.11 \\
WC $(\mathrm{cm})$ & Sig (2-tailed) & 0.43 & 0.13 & 0.41 \\
& N & 60 & 60 & 60 \\
& Pearson correlation & -0.23 & 0.25 & 0.18 \\
SAD $(\mathrm{cm})$ & Sig (2-tailed) & 0.08 & 0.06 & 0.18 \\
& N & 60 & 60 & 60 \\
& Pearson correlation & $-0.26^{*}$ & 0.81 & $0.26^{*}$ \\
WHR & Sig (2-tailed) & 0.05 & 0.17 & 0.05 \\
& N & 60 & 60 & 60 \\
& Pearson correlation & -0.09 & $0.29^{*}$ & 0.21 \\
WTH & Sig (2-tailed) & 0.50 & 0.03 & 0.12 \\
& N & 60 & 60 & 60 \\
& Pearson correlation & -0.18 & 0.13 & 0.14 \\
$\%$ BF & Sig (2-tailed) & 0.90 & 0.31 & 0.30 \\
& N & 60 & 60 & 60 \\
\hline
\end{tabular}

*Correlation is significant at the 0.05 level (2-tailed)

Table 8. Relationship of Physical Attributes with Distance Covered by the Diabetics during 6-Minutes Walk Test.

\begin{tabular}{llll}
\hline & & Dist (m) & PR2 (beat / min) \\
\hline \multirow{3}{*}{ Weight $(\mathrm{kg})$} & Pearson correlation & $-0.28^{*}$ & $0.37^{* *}$ \\
& Sig (2-tailed) & 0.03 & 0.01 \\
& $\mathrm{~N}$ & 60 & 60 \\
& Pearson correlation & $-0.29^{*}$ & $0.32^{*}$ \\
$\mathrm{BMI}(\mathrm{kg} / \mathrm{m} 2)$ & Sig (2-tailed) & 0.03 & 0.02 \\
& $\mathrm{~N}$ & 60 & 60 \\
& Pearson correlation & -0.20 & $0.28^{*}$ \\
$\% \mathrm{BF}$ & Sig (2-tailed) & 0.13 & 0.03 \\
& $\mathrm{~N}$ & 60 & 60 \\
\hline
\end{tabular}

*Correlation is significant at the 0.05 level (2-taled)

*Correlation is significant at the 0.01 level (2-taled)

\section{Discussion}

The intensity of exercise is a key determinant of how an individual may respond to a given exercise regimen in terms of improvements in glucose tolerance [13]. If the exercise intensity is too low, the maximal benefits of exercise would not be realized. Anthropometric variable which is physical measurable attributes of man could be related to risk of developing health problem. Wei et al reported after conducting a study among Mexican Americans aged 25 to 64 years that waist circumference had the highest correlation to development of non-insulin dependent diabetes mellitus among other anthropometric variables, such as BMI, HC, WHR, and WTH [7]. Also, Ohrvall et al. conducted their study in Uppsala, Sweden, and found SAD to have stronger correlation with cardiovascular risk and with other risk factors in the metabolic syndrome than WC, WHR and BMI [9]. The result of this study showed that the BMI, WC, SAD, WHT, and \% body fat of diabetic were significantly higher than that of non-diabetic participants. This result is in line with the report of Wei et al. among Mexican Americans and that of Ohrvall et al. in Uppsala Sweden [7, 9]. The result of this study, however, disagreed with that of Dalton et al. who reported that WHR was the best indicator of obesity out of the anthropometric variables tested [8].

Correlation test of some anthropometric variables to cardiovascular risk factors in this study showed that BMI WHR and WTH had significant correlation with blood pressure and fasting blood glucose of the diabetics. BMI showed positive correlation with systolic blood pressure, meaning that the higher the BMI, the higher the systolic blood pressure. This is in line with Thomas et al. [13]. They reported that there was a continuous relationship between increasing obesity and cardiovascular risk [13]. WHR had negative correlation with FBS, indicating the lower the WHR the higher the FBS. Contrarily, previous report was that the higher the waist to hip ratio, the more masculine the pattern of adipose tissue distribution and the grater the risk of diseases such as non-insulin dependent diabetes mellitus. The reason for the disagreement may be due to the fact that other health problem could as well lead to diabetes in addition to predisposing ability of the anthropometric variables.

WHR also showed positive correlation with diastolic blood pressure. This is in agreement with the report of Dalton et al. that WHR had the strongest correlations with CVD [8]. WTH had positive correlation with systolic blood pressure. WTH is one of the anthropometric indexes in the prediction of hypertension, Type 2 diabetes mellitus and dyslipidaemia in a Mexican population. It seems that BMI, WHR and WTH have about the same level of relationship in determining the development of cardiovascular risk factors as indicated in this study WC and SAD however showed no significant relationship to cardiovascular risk factors. There was no significant difference between anthropometric variables of male and female diabetic in this study except height. This indicates that anthropometric variables of male and female diabetics are similar.

Diabetes had been identified as one of the risk factors for the development of cardiovascular disease [1]. Mortality from cardiovascular diseases has been reported to also increase two to four folds in diabetics when compared to the general population $[4,5]$. Cardiovascular complications that arise as a result of type 2 diabetes may be due to effect of body's (especially muscle including heart's muscle) inability to maximally metabolize glucose for energy demand of the body, thereby placing excessive stress on the heart. This may eventually lead to cardiovascular disease. The result of this study showed significant difference between diabetic and non-diabetic cardiovascular parameters. The mean values of fasting blood sugar, systolic blood pressure, diastolic blood pressure, were higher than non-diabetics. Also the significant differences in the cardiovascular parameters seen in the systolic blood pressure, diastolic blood pressure and pulse rate were observed at both the pre and post endurance exercise. The result of this study therefore indicated higher cardiovascular risk in the diabetics than non diabetics. This is in agreement with the submission of Stewart et al. and; that of Robinson and Johnson [4,5].

Comparison between male and female diabetics, showed 
no significant difference in the cardiovascular variables. The distance covered by the non diabetic during six minute walk test was longer than distance covered by the diabetic despite higher response of measured cardiovascular factors during the sub maximal endurance work. The correlation test of weight, $\mathrm{BMI}$ and $\% \mathrm{BF}$ with distance covered showed negative significant relationship in the case of weight and BMI. This indicates that the higher the weight and BMI the less the distance covered. It is a general fact that obese people walk less. Weight, BMI and \% BF, showed positive correlation with post exercise pulse rate. This means that the higher the body weight, $\mathrm{BMI}$ and $\% \mathrm{BF}$, the higher the postexercise pulse rate. This shows that the non diabetics were more fit than the diabetics.

This study concluded that there was significant relationship between BMI, WHR; and with blood pressure and blood glucose. Similarly, there was significant difference in most of the anthropometric valuables (BMI, $\mathrm{WC}, \mathrm{WTH}, \mathrm{SAD}, \% \mathrm{BF}$ ) tested between diabetics and nondiabetics. Also, the selected cardiovascular parameters tested pre and post exercise showed significant difference between diabetics and non-diabetics. Both male and female diabetics had similar anthropometric and cardiovascular variables.

\section{References}

[1] Manolis, A.J. (2005). Exercise and Hypertension. European Society of Hypertension Scientific Newsleter.

[2] American Heart Association (1999). Cardiovascular fitness: 1999 Heart and Stroke Update.

[3] Adler, A.I., Strafton, I.M; Neil, H.A. W; Yudkin, J.S; Mattews, D.R; Cull, C.A; et al (2000). Association of Systolic Blood Pressure With Macrovascular and Microvascular Complications of Type 2 Diabetes (UKPDS 36): Prospective Observational Study. BMJ 321 (7258), 412.

[4] Stewart, M.W; Laker, M.P; \& Alberti; K.G.M.M. (1993). Effect of Self Monitoring of Triglyceride Concentration In Non-Insulin Dependent Diabetes. BMJ, 306 ( 6876).

[5] Robinson, S. \& Johnson, D.G. (1997). Metabolic Disorder: Diabetes. In S. Tomlinson, A.M. Heagerty \& A.P. Weetman (Eds.), Mechanisms of Disease: An Introduction to Clinical Science, ( PP 202-244), Cambridge: Cambridge University Press
[6] Van ltallie T.B. (1988). Topography of Body fat: Relationship to Risk of Cardiovascular and Other Diseases. Tn T.G. Lohman, A.T; Roche \& R. Martorell (Eds.), Anthropometric Standardization Reference Manual, (PP 143-149), Champaign: Human Kinetics Book.

[7] Wei, M; Gaskill, S.P; Haffner, S.M. \& Stern, M.P. (1997). Waist Circumference as The Best Predictor of Non-Insulin Dependent Diabetes Mellitus (NIDDM) Compared to Body Mass Index, Waist/Hip Ratio and Other Anthropometric Measurements In Mexican Americans - A 7-year Prospective Study. Retrieved May 4, 2007 from http://www.ncbi.nlm.nih.gov /entrezl query.fcgi?cmd=retrieve $\& \mathrm{~d} b=$ pu bmed.

[8] Dalton, M; Cameron, A.J; Zimmet, P.Z; Shaw, J.E; Jolley, D; Dunstan, D.W; et al. (2003). Waist Circumference, Waist-Hip Ratio and Body Mass Index and Their Correlation With Cardiovascular Disease Risk in Australian Adult. Journal of Intern Medicine. Retrieved May 4, 2007 from $\mathrm{http}: / /$ www.ncbi.nih.gov/entrez/query.fegi? $=$ retriveve $\& \mathrm{db}=\mathrm{pu}$ bmed\&dopt=citattion\&list_uids.

[9] Ohrvall, M. Berghund, L. \& Vessby, B. (2000). Saggital Abdominal Diameter Compared With Other Anthropometric Measurements In Relation to Cardiovascular Risk. International Journal of Obesity. 24 (4). Retrieved May 10,2007 from http://www.nature.com/ijo/journal/v24/n4/full/0801 186a.html.

[10] Thomas, G.N; HO, S; Lam, K.S.L; Janus, E.D; Hedley, A.J \& Lam, T.H. (2004). Impact of Obesity and Body Fat Distribution on Cardiovascular Risk factor In Hong Kong Chinese. Obesity Research, 12, 1805-1813. Retrieved May 10, 2007 from http://www .obesityresearch.org/cgi/content/full/12/11/1805.

[11] Nayak, B.S. \& Roberts, L. (2006). Relationship Between Inflammatory Markers, Metabolic and Anthropometric Variables In The Caribbean Type 2 Diabetic Patients With and Without Microvascular Complications. Journal of Inflammation. 3:17.

[12] McAlister, F.A. and Strans, S.E. (2001). What Is This Person's Blood Pressure? In C.D. Mulrow (Ed.), Evidence-based Hypertension, ( PP 9-32), London: BMJ Books.

[13] Wright, D.C; \& Swan, P.O. (2001). Optimal Exercise Intensify For Individuals With Impaired Glucose Tolerance. American Diabetics Association, 14,93-97. 